\title{
Endogenously synthesized n-3 fatty acids in fat-1 transgenic mice prevent melanoma progression by increasing E-cadherin expression and inhibiting $\beta$-catenin signaling
}

\author{
XUAN YIN ${ }^{1}$, XIONG-WEI YU ${ }^{1}$, PAN ZHU ${ }^{1}$, YUAN-MING ZHANG ${ }^{1}$, \\ XIAO-HONG ZHANG ${ }^{1}$, FENG WANG ${ }^{2}$, JIN-JIE ZHANG ${ }^{3}$, WANG YAN ${ }^{4}$, YANG XI ${ }^{1}$, \\ JIAN-BO WAN ${ }^{5}$, JING-XUAN KANG ${ }^{6}, Z^{2}-Q U A N Z^{1}{ }^{1 *}$ and SHI-ZHONG BU ${ }^{1 *}$

\begin{abstract}
${ }^{1}$ Medical School, Ningbo University, Ningbo, Zhejiang 315211; ${ }^{2}$ Clinical Laboratory, Lihuili Hospital, Ningbo, Zhejiang 315040;
${ }^{3}$ Maritime Faculty, Ningbo University, Ningbo, Zhejiang 315211; ${ }^{4}$ Neurosurgery Department, Second Hospital of Ningbo, Ningbo, Zhejiang 315010; ${ }^{5}$ State Key Laboratory of Quality Research in Chinese Medicine, Institute of Chinese Medical Sciences, University of Macau, Macau, SAR 519000, P.R. China; ${ }^{6}$ Laboratory for Lipid Medicine and Technology,
\end{abstract} \\ Massachusetts General Hospital and Harvard Medical School, Boston, MA 02114, USA
}

Received September 20, 2015; Accepted July 13, 2016

DOI: $10.3892 / \mathrm{mmr} .2016 .5639$

\begin{abstract}
Malignant melanoma is the most lethal form of skin cancer. Although preclinical studies have shown that n-3 polyunsaturated fatty acids (PUFAs) are beneficial for prevention of melanoma, the molecular mechanisms underlying the protective effects of n-3 PUFAs on melanoma remain largely unknown. In the present study, endogenously increased levels of n-3 PUFAs in the tumor tissues of omega-3 fatty acid desaturase (fat-1) transgenic mice was associated with a reduction in the growth rate of melanoma xenografts. This reduction in tumor growth in fat-1 mice compared with wild-type controls may have been associated, in part, to the: i) Increased expression of E-cadherin and the reduced expression of its transcriptional repressors, the zinc finger E-box binding homeobox 1 and snail family transcriptional repressor 1; ii) significant repression of the epidermal growth factor receptor/Akt/ $\beta$-catenin signaling pathway; and iii) formation of significant levels of $n-3$ PUFA-derived lipid mediators, particularly resolvin D2 and E1, maresin 1 and 15-hydroxyeicosapentaenoic acid. In addition, vitamin E administration counteracted n-3 PUFA-induced lipid peroxidation and enhanced the antitumor effect of $n-3$ PUFAs, which suggests that the protective role of n-3 PUFAs against melanoma is not mediated by n-3 PUFAs-induced lipid peroxidation. These results highlight a potential role of
\end{abstract}

Correspondence to: Dr Zu-Quan Zou or Professor Shi-Zhong Bu, Medical School, Ningbo University, 818 Fenghua Road, Ningbo, Zhejiang 315211, P.R. China

E-mail: zouzuquan@nbu.edu.cn

E-mail: bushizhong@nbu.edu.cn

${ }^{*}$ Contributed equally

Key words: fat-1, E-cadherin, $\beta$-catenin, lipid mediator, melanoma n-3 PUFAs supplementation for the chemoprevention of melanoma in high-risk individuals, and as a putative adjuvant agent in the treatment of malignant melanoma.

\section{Introduction}

Malignant melanoma is the most aggressive form of cutaneous malignancy, and accounts for $\sim 3 \%$ of all cases of malignant tumor (1). The global incidence of melanoma has been rising, including an annual increase of between 3 and $7 \%$ for Caucasians in developed countries (2). Although melanoma accounts for only $4 \%$ of all skin cancer cases, it is responsible for $\sim 80 \%$ of all skin cancer-related cases of mortality due to its extreme aggressiveness and resistance to current therapeutic agents (3). To date, a number of promising targeted agents, including the B-Raf inhibitors vemurafenib and dabrafenib, are undergoing or have completed phase III clinical trials; however, the majority of these have been demonstrated to be ineffective and the 5-year survival rate of the vast majority of patients diagnosed with metastatic melanoma is <5\% (4). Therefore, novel treatment strategies are required for patients with metastatic melanoma.

Malignant transformation is often characterized by major changes in the organization of the cytoskeleton, decreased cell-to-cell adhesion and increased cell motility. The loss of the cellular adhesion molecule and single-span transmembrane glycoprotein, E-cadherin, is positively associated with tumor invasiveness, metastatic dissemination and poor patient prognosis (5). E-cadherin is expressed in epithelial cells, and intercellular homophilic interactions with E-cadherin expressed by neighboring cells, leads to the formation of cell-to-cell adherens junctions (AJs) and a tight polarized cell layer (6). Loss of E-cadherin expression triggers epithelial-to-mesenchymal transition (EMT), which provides cancer cells with increased motility and invasiveness (7). Clinical studies have shown that the majority of patients with melanoma treated with targeted B-Raf proto-oncogene, 
serine/threonine kinase inhibitors, such as vemurafenib and dabrafenib, develop resistance to these therapies after only a several months due to decreased E-cadherin expression in the tumor tissues of patients (8). $\beta$-Catenin, the principal effector of the Wnt signaling cascade, serves a crucial role in morphogenesis and human cancer through its dual function in mediating cell-to-cell AJs, and as a signaling molecule in numerous signaling pathways (9). $\beta$-catenin forms an AJ complex with E-cadherin through binding to the intracellular domain of E-cadherin, which sequesters it at the plasma membrane and hinders its entry into the nucleus (10). Numerous studies have demonstrated that $\beta$-catenin signaling is implicated in melanoma progression and metastasis $(11,12)$. In recent years, a number of plant-derived natural products have been described as potential alternative therapies for metastatic melanoma, that function by increasing E-cadherin expression and by disrupting the $\beta$-catenin signaling pathway (13). For instance, catechins, a class of flavonoids, exhibit anti-melanoma activity through the restoration of E-cadherin and the suppression of $\mathrm{N}$-cadherin expression levels (14). In addition, the flavonoid complex silymarin, blocks the invasion of melanoma cells by inhibiting $\beta$-catenin translocation (15). Therefore, inhibiting the proliferative, migratory and invasive capacity of cancer cells with chemopreventative dietary factors, may provide a potential strategy for melanoma prevention and/or treatment.

Among the dietary factors, n-3 polyunsaturated fatty acids (PUFAs) have gained attention as potential preventative and/or adjuvant agents in the treatment of malignant melanoma (16). A number of preclinical in vitro and in vivo studies have attempted to clarify the possible mechanisms underlying the proposed anticancer effects of eicosapentaenoic acid (EPA) and docosahexaenoic acid (DHA), which are the two principal n-3 PUFAs found in fish and seafood $(17,18)$. A number of published preclinical and epidemiological studies provide evidence to suggest that dietary or exogenously derived fatty acids may serve a vital role in the development and progression of malignant melanoma $(19,20)$; however, conflicting results and discrepancies between studies preclude definitive conclusions $(21,22)$. For instance, Kirkpatrick et al (23) reported no association between the consumption of PUFAs and melanoma risk, whereas, Salem et al (24) demonstrated that fish oil supplementation of B16 melanoma-bearing mice enhanced melanoma cell growth and metastases and decreased mouse survival rates. These conflicting results reflect numerous confounding dietary elements. Indeed, fish and corn oil, which are generally used to investigate the effects of the ratio of n-3/n-6 PUFAs on disease development and progression, consist not only of n-3 and n-6 PUFAs, but also additional fatty acids and lipid soluble vitamins (25).

In the present study, the potential of high n-3 PUFAs supplementation in the prevention of melanoma cell growth was investigated using omega- 3 fatty acid desaturase (fat-1) transgenic mouse xenografts, which carry the fat- 1 gene from the Caenorhabditis elegans roundworm (26). This gene, which is not present in mammals, encodes an n-3 PUFA desaturase that catalyzes the conversion of n-6 to n-3 PUFAs. Therefore, the fat-1 transgenic mice were endogenously enriched with n-3 PUFAs and were characterized by a lower ratio of n- $6 / n-3$ PUFAs compared with their wild-type (WT) littermates, by using a single diet high in n-6 PUFAs. The need for dietary n-3
PUFAs supplementation was eliminated and corresponding confounding factors of diet were removed. Therefore, the fat-1 transgenic mouse model is ideal for addressing the effects of the tissue n-6/n-3 fatty acid ratio in melanoma tumorigenesis.

Endogenous n-3 PUFAs have been previously reported to exert their antitumor effects in melanoma through the prostaglandin (PG) E3-mediated activation of the phosphatase and tensin homologue deleted on chromosome 10 signaling pathway in fat-1 transgenic mice injected subcutaneously with melanoma B16-F0 cells (27). However, whether n-3 PUFA-derived lipid mediators and the E-cadherin $/ \beta$-catenin signaling pathway are associated with n-3 PUFA-mediated antitumor effects in malignant melanoma remains largely unknown. In the present study, B16-F10 mouse melanoma cells, which have a higher metastatic potential compared with B16-F0 melanoma cells, were injected into fat-1 transgenic and WT mice in order to evaluate its tumorigenicity. The results demonstrated that increased E-cadherin expression, inhibition of the $\beta$-catenin signaling pathway and biosynthesized n-3 PUFA-derived bioactive mediators, are involved in the antitumor effects of n-3 PUFAs in melanoma.

\section{Materials and methods}

Cells and reagents. The murine B16-F10 melanoma cell line (American Type Culture Collection, Manassas, VA, USA) was maintained in a humidified incubator at $37^{\circ} \mathrm{C}$ and $5 \% \mathrm{CO}_{2}$ in Dulbecco's modified Eagle's medium (DMEM; Gibco; Thermo Fisher Scientific, Inc., Waltham, MA, USA), supplemented with $10 \%$ fetal bovine serum. The primary antibodies, mouse anti-E-cadherin (catalog no. 14472), mouse anti-N-cadherin (catalog no. 14215), rabbit anti- $\beta$-catenin (catalog no. 9582), rabbit anti-zinc finger E-box 1 (ZEB1; catalog no. 3396), rabbit anti-Snail (catalog no. 3879), rabbit anti-Slug (catalog no. 9585), rabbit anti-c-Myc (catalog no. 13987), rabbit anti-Akt (pan; catalog no. 4685), rabbit anti-phosphorylated (p)-Akt (Thr308; catalog no. 13038), rabbit anti-nuclear factor

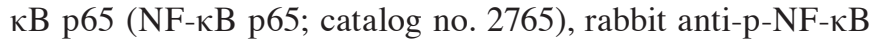
p65 (Ser536; catalog no. 3033), rabbit anti-glycogen synthase kinase-3 $\beta$ (GSK-3 $\beta$; catalog no. 9315), rabbit anti-p-GSK-3 $\beta$ (Ser9; cataolog no. 9322), rabbit anti-signal transducer and activator of transcription 3 (STAT3; catalog no. 12640), rabbit anti-p-STAT3 (Tyr705; catalog no. 9145), rabbit anti-epidermal growth factor receptor (EGFR; catalog no. 4267), rabbit anti-GAPDH (catalog no. 5174) and the horseradish peroxidase-linked secondary antibodies, anti-rabbit IgG (catalog no. 7074) and anti-mouse IgG (catalog no. 7076) were purchased from Cell Signaling Technology, Inc. (Danvers, MA, USA). The primary mouse anti-twist (catalog no. ab175430) and rabbit anti-smad interacing protein 1 (ZEB2; catalog no. ab138222) antibodies were obtained from Abcam (Cambridge, MA, USA). Primary and secondary antibodies were used at dilutions of 1:1,000 and 1:4,000, respectively. Vitamin E (Vit E) powder was purchased from Tianjin Jianfeng Natural Product R\&D Co., Ltd. (Tianjin, China). Chloral hydrate was purchased from Shanghai Meilian Biotechnology Co., Ltd. (Shanghai, China).

Animals and diet. The fat-1 transgenic mice were donated by Dr Jing X. Kang at Massachusetts General Hospital and 
Harvard Medical School (Boston, MA, USA). A total of 13 male heterozygous fat-1 mice were bred with 26 WT C57BL/6 female mice (26). Each mouse was genotyped and phenotyped by polymerase chain reaction and gas chromatography using isolated DNA and total lipids from mice tails, respectively. Specific pathogen-free transgenic and WT animals were fed a $10 \%$ safflower oil diet, and housed in standard cages in temperature under humidity-controlled conditions with a 12-h light/dark cycle. At 10-12 weeks of age, heterozygous fat-1 male mice and nontransgenic littermate controls were used for the purposes of the present study. Their diets (per $100 \mathrm{~g}$ ) consisted of $4.5 \mathrm{~g}$ sucrose, $18.6 \mathrm{~g}$ casein, $8.6 \mathrm{~g}$ cellulose, $50 \mathrm{~g}$ wheat starch, $0.3 \mathrm{~g}$ DL-methionine, $7 \mathrm{~g}$ mineral mix, $1 \mathrm{~g}$ vitamin mix and $10 \mathrm{~g}$ safflower oil. A total of 10 fat- 1 and $10 \mathrm{WT}$ mice were assigned to Vit E groups and another 10 fat-1 and $10 \mathrm{WT}$ mice were assigned to negative control groups. The Vit E-treatment group was administered with $100 \mathrm{IU} / \mathrm{kg}$ Vit E (dissolved in sterile water) via oral gavage for 3 weeks. Sterile water was administered in the same manner to the control group. All animal experiments were conducted in accordance with the guidelines for the use and care of laboratory animals, and approved by the Ethical Committee of Ningbo University (Ningbo, China).

Cell injections and tumor measurement. Cultured B16-F10 melanoma cells were collected by trypsin digestion $(0.05 \%$ trypsin-EDTA) for 2 min and washed twice with DMEM before the cell number was ascertained. Each male mouse was injected subcutaneously with $2.5 \times 10^{6}$ cells suspended in $100 \mu \mathrm{l}$ DMEM into the area overlying the abdomen. The day of the injection of B16-F10 cells was designated day 0 . Tumor volume was assessed by conducting caliper measurements every 2 days, and calculated according to the following formula: Tumor volume $=$ Shortest $\operatorname{diameter}^{2} \mathrm{x}$ the largest diameter x 0.5. Following 15 days of cell injection, the mice were anesthetized with an intraperitoneal injection of $350 \mathrm{mg} / \mathrm{kg}$ chloral hydrate and sacrificed by exsanguination. The tumors were removed and stored at $-80^{\circ} \mathrm{C}$ for downstream analyses.

Analysis of fatty acids and lipid mediators. Fatty acid composition was determined using gas chromatography (GC) as described previously (28). Briefly, tissues were ground to a powder form using liquid nitrogen, and the methyl esters of the fatty acids from the lipid extract were transesterified with $\mathrm{H}_{2} \mathrm{SO}_{4}$ in methanol (5\%, v/v), together with toluene, in sealed tubes at $70^{\circ} \mathrm{C}$ for $2 \mathrm{~h}$. Fatty acid methyl esters were analyzed using the Shimadzu GC-14C gas chromatograph (Shimadzu Co., Kyoto, Japan) equipped with a flame-ionization detector and a $60 \mathrm{~m} \times 0.25 \mathrm{~mm}$ (internal diameter) $\times 0.25 \mu \mathrm{m}$ (film thickness) fused silica bonded phase column (DB-23; Agilent Technologies, Inc., Santa Clara, CA, USA). The fatty acid methyl esters were identified by comparing sample mixtures with standard mixtures of fatty acid methyl esters (Nu-Chek Prep, Inc., Elysian, MN, USA). A $1 \mu l$ aliquot of each sample was injected onto the column. Nitrogen was the carrier gas and the flow rate was $4 \mathrm{ml} / \mathrm{min}$. The injector and detector temperature was $270^{\circ} \mathrm{C}$. The column temperature was $180^{\circ} \mathrm{C}$ and held for $10 \mathrm{~min}$; the temperature was then increased to $200^{\circ} \mathrm{C}$ at a rate of $20^{\circ} \mathrm{C} / \mathrm{min}$ and held for $10.5 \mathrm{~min}$; the temperature was further increased to $215^{\circ} \mathrm{C}$ at a rate of $20^{\circ} \mathrm{C} / \mathrm{min}$ and held for $5 \mathrm{~min}$; finally, it was increased to $215^{\circ} \mathrm{C}$ at a rate of $20^{\circ} \mathrm{C} / \mathrm{min}$ and held for $4.75 \mathrm{~min}$. Quantification of sample fatty acid compositions was achieved by comparing peak areas with the nonadecanoic acid internal standard (Sigma-Aldrich, St. Louis, MO, USA), which was added to the samples (1 $\mathrm{mg}$ internal standard/500 mg sample) prior to extraction. The composition of fatty acids was expressed as the relative percentage of the total fatty acids according to their peak areas.

Lipid mediators from n-3 and n- 6 fatty acids were identified using liquid chromatography (LC)-tandem mass spectrometry (MS) methods as described previously (29). In brief, each tumor sample was homogenized in $2 \mathrm{ml} 15 \% \mathrm{v} / \mathrm{v}$ ice-cold methanol. The internal standards PGB2- $\mathrm{d}_{4}, \mathrm{PGD} 2-\mathrm{d}_{4}$, 15-HETE- $\mathrm{d}_{8}$ and resolvin (RV) E1- $\mathrm{d}_{4}(20 \mathrm{ng}$ per sample each) were added to each sample and n-3 and n-6 fatty acid mediators were extracted using Strata-X reversed-phase SPE columns (8B-S100-UBJ; Phenomenex, Torrance, CA, USA). An Acquity ultra performance (UP) LC system (Waters Corporation, Milford, MA, USA) was used. Reversed-phase separation was performed on an Acquity UPLC BEH shield RP18 column [2.1 $100 \mathrm{~mm}$ (internal diameter); $1.7 \mu \mathrm{m}$ (film thickness); Waters Corporation]. The mobile phase consisted of (A) acetonitrile/water/acetic acid (60/40/0.02\% v/v) and (B) acetonitrile/isopropenyl acetate $(50 / 50 \% \mathrm{v} / \mathrm{v})$. Gradient elution was carried out for $5 \mathrm{~min}$ at a flow rate of $0.5 \mathrm{ml} / \mathrm{min}$. Gradient conditions were as follows: $0-4.0 \mathrm{~min}, 0.1-55 \% \mathrm{~B}$; 4.0-4.5 min, 55-99\% B; 4.5-5.0 min, 99\% B. A $10 \mu \mathrm{l}$ aliquot of each sample was injected onto the column. The column temperature was maintained at $40^{\circ} \mathrm{C}$. Mass spectrometry was performed on an ABI/Sciex 6500 QTRAP hybrid, triple quadrupole, linear ion trap mass spectrometer (AB Sciex, Framingham, MA, USA) equipped with a Turbo V ion source. Lipid mediators were detected in negative electrospray ion mode. Curtain gas, nebulizer gas and turbo-gas were set at 10,30 and $30 \mathrm{psi}$, respectively. The electrospray voltage was $-4.5 \mathrm{kV}$, and the turbo ion spray source temperature was $525^{\circ} \mathrm{C}$, and a methanol:water:acetate $(60: 40: 0.02 \%)$ mobile phase was used with a $0.5 \mathrm{ml} / \mathrm{min}$ flow rate. The following multiple reaction monitoring transitions were used: PGE2 $\mathrm{m} / \mathrm{z}$ 351>271, PGE3 m/z 349>269, 12-hydroxyeicosapentaenoic acid (HEPE) $\mathrm{m} / \mathrm{z} 317>179,15$-HEPE $\mathrm{m} / \mathrm{z} 317>219$, RVD $2 \mathrm{~m} / \mathrm{z}$ 375>175, RVE1 $\mathrm{m} / \mathrm{z}, 349>195$ and Maresin $1 \mathrm{~m} / \mathrm{z}$ 359>177.

Western blot analysis. Tumor tissues (30 mg) were homogenized in Triton-X protein lysis buffer $(20 \mathrm{mM}$ Tris- $\mathrm{HCl}, 1 \mathrm{mM}$ EDTA, $140 \mathrm{mM} \mathrm{NaCl}, 1 \%$ NonidetP-40, $1 \%$ aprotinin, $1 \mathrm{mM}$ phenylmethylsulfonyfluoride and $1 \mathrm{mM}$ sodium vanadate; $\mathrm{pH}$ 7.6). The sample protein concentration was determined using a bicinchoninic acid protein assay. Aliquots of protein $(50 \mu \mathrm{g})$ were fractionated by $10 \%$ SDS-PAGE and transferred onto nitrocellulose membranes. After nonspecific binding sites were blocked with 5\% nonfat milk in Tris-buffered saline, blots were incubated overnight at $4^{\circ} \mathrm{C}$ with primary antibodies, and then incubated for $1 \mathrm{~h}$ at room temperature with horseradish peroxidase-conjugated secondary antibodies. Reactive protein bands were analyzed using enhanced chemiluminescence .

Lipid peroxidation analysis. Lipid peroxidation in urine samples was determined using an malondialdehyde (MDA) 
assay kit (Beyotime Institute of Biotechnology, Haimen, China) according to the manufacturer's instructions. Thiobarbituric acid (TBA; $200 \mu \mathrm{l}$ ) reagent was added to $100 \mu 1$ urine sample. The reaction mixture was subsequently incubated in a water bath at $100^{\circ} \mathrm{C}$ for $15 \mathrm{~min}$. After cooling to room temperature, the mixture was centrifuged at $1,000 \mathrm{x} \mathrm{g}$ for $10 \mathrm{~min}$ at room temperature and the supernatant was separated, before the absorbance was read at $532 \mathrm{~nm}$. The concentration of MDA was determined using a standard curve.

Statistical analysis. For each treatment group, data were presented as the arithmetical mean \pm standard error. Statistical significance between groups was determined using the Student's $t$-test. $\mathrm{P}<0.05$ and $\mathrm{P}<0.01$ was considered to indicate a statistically significant difference.

\section{Results}

n-3 PUFAs inhibited the tumorigenicity of B16-F10 melanoma cells in fat-1 transgenic mice. In order to test the hypothesis that a balanced n-6/n-3 fatty acid ratio in fat- 1 transgenic mice is associated with a decreased risk of melanoma, B16-F10 murine melanoma cells were injected into transgenic fat-1 and WT mice, and the tumorigenicity of inoculated tumor cells was examined. As shown in Fig. 1, a marked difference in the tumor growth rate was observed between fat-1 transgenic $(n=10)$ and WT mice $(n=10)$. Over an observation period of 15 days, all mice initially developed a palpable tumor by day 7. The tumors in fat- 1 transgenic mice exhibited a markedly slower growth rate when compared to those in WT mice.

Fatty acid profiles in the tumor tissues of fat-1 and WT mice. Analysis of the total lipids extracted from the tumor tissues of fat-1 and WT mice revealed distinct lipid profiles between the two groups (Fig. 2). Compared with WT mice, fat-1 transgenic mice displayed significantly increased levels of $n-3$ fatty acids, including 20:5n-3 (EPA; $\mathrm{P}=0.0441), 22: 5 n-3$ [docosapentaenoic acid (DPA; $\mathrm{P}=0.0482)$ and 22:6n-3 (DHA; $\mathrm{P}=0.0151$ ), and exhibited decreased concentrations of $n-6$ fatty acids, including 18:2n-6 (linoleic acid), 20:3n-6 [dihomo-gamma-linolenic acid (DGLA)], 20:4n-6 [arachidonic acid (AA)] and 22:4n-6 (adrenic acid) in the tumor tissues (Fig. 2A). In particular, the levels of linoleic acid $(\mathrm{P}=0.0356)$ and $\mathrm{AA}(\mathrm{P}=0.0122) \mathrm{n}-6$ fatty acids were significantly reduced in tumors from fat-1 mice compared with WT animals. As shown in Fig. 2B the $\mathrm{n}-6 / \mathrm{n}-3$ fatty acid ratio in tumors from fat-1 mice $(7.7 \pm 1.6)$ was significantly lower than that of WT mice $(22.0 \pm 3.1 ; \mathrm{P}=0.0097)$, despite the animals being fed the same diet. These results indicate the fat- 1 transgene is functionally active in vivo, and endogenously catalyzes the conversion of n-6 to n-3 PUFAs.

Lipid mediators derived from AA (prostanoids, leukotrienes, lipoxins and epoxyeicosatrienoic acids), EPA (E-series resolvins) and DHA (D-series resolvins, protectins and maresins) exert their potent actions through the promotion or resolution of inflammation (30). Therefore, the levels of n-6 and n-3 PUFA-derived lipid mediators in the melanoma tumor tissues of WT and fat-1 transgenic mice were determined using UPLC-UV-tandem MS analysis, in order to determine whether the observed difference in tumor growth between these two groups was associated with differences in the levels of lipid
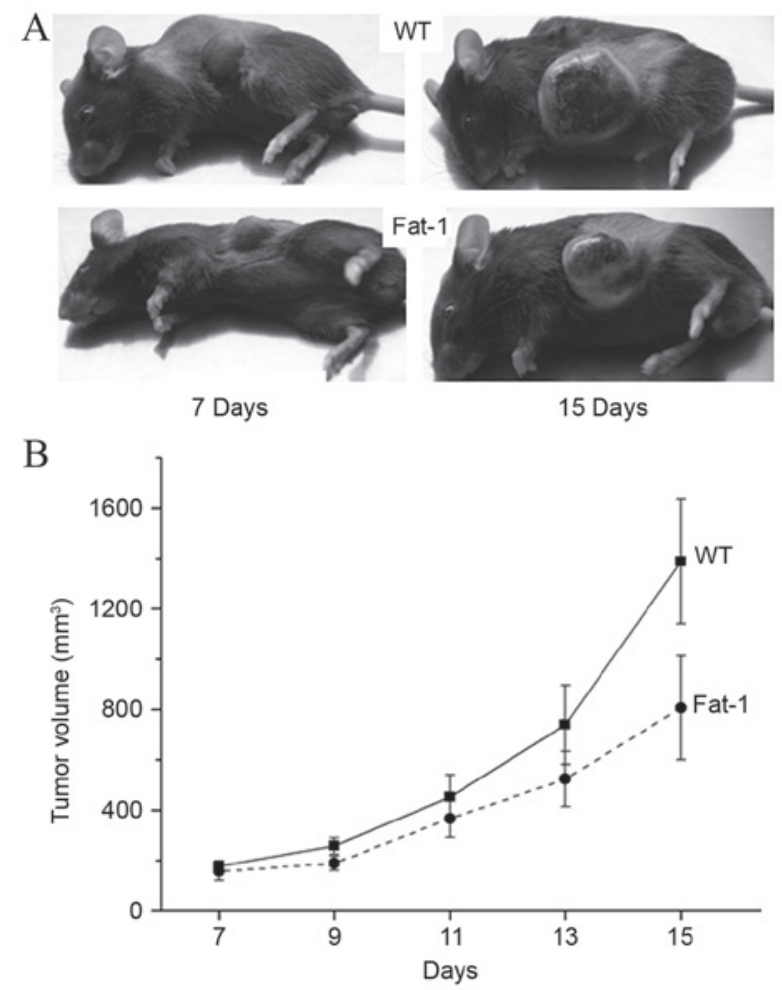

Figure 1. Effect of fat-1 gene expression on the tumorigenicity of B16-F10 melanoma cells in vivo. B16-F10 cells (2.5x106) were injected subcutaneously into 10 male, 12-week-old fat-1 transgenic and $10 \mathrm{WT}$ mice of the same age. (A) Representative photographs showing tumor formation at 7 and 15 days post-injection in WT and fat- 1 transgenic mice. (B) The growth rates of melanoma tumors in WT and fat-1 transgenic mice. Data points represent the mean \pm standard error. Fat-1, omega-3 fatty acid desaturase; WT, wild-type.

mediators. As shown in Fig. 3, a significant increase in the levels of fatty acid metabolites derived from EPA, [12-HEPE $(\mathrm{P}=0.0187), 15-\mathrm{HEPE}(\mathrm{P}=0.0224), \mathrm{PGE} 3(\mathrm{P}=0.0320)$ and RVE1 $(\mathrm{P}=0.0098)$ and DHA $(\mathrm{RVD} 2(\mathrm{P}=0.0092)$ and Maresin1 $(\mathrm{P}=0.042))$ were observed in tumors from fat-1 mice compared with WT controls. In contrast, no significant difference in the levels of proinflammatory PGE2 was observed between fat-1 and WT mice.

Differential protein expression levels of E-cadherin and its master regulators in fat-1 transgenic and WT mice. In order to determine whether fat-1 expression, and the associated increase in n-3 PUFA levels, affects E-cadherin and $\mathrm{N}$-cadherin expression levels, melanoma tumor tissues from transgenic fat-1 mice and WT controls were analyzed for the expression of E-cadherin and N-cadherin by western blotting. As shown in Fig. 4, the protein expression levels of E-cadherin and $\mathrm{N}$-cadherin were markedly upregulated and downregulated in the tumor tissues of fat- 1 mice compared with those of the WT controls, respectively. This implies that E-cadherin and $\mathrm{N}$-cadherin expression may be modulated by n-3 PUFAs. Several transcription factors, including Snail, Slug, ZEB1, ZEB2 and Twist, function as transcriptional repressors of E-cadherin (31). Therefore, western blotting was used to investigate whether the observed increase in E-cadherin expression levels in the melanoma tissues of fat-1 mice may be mediated by alterations in the protein expression 

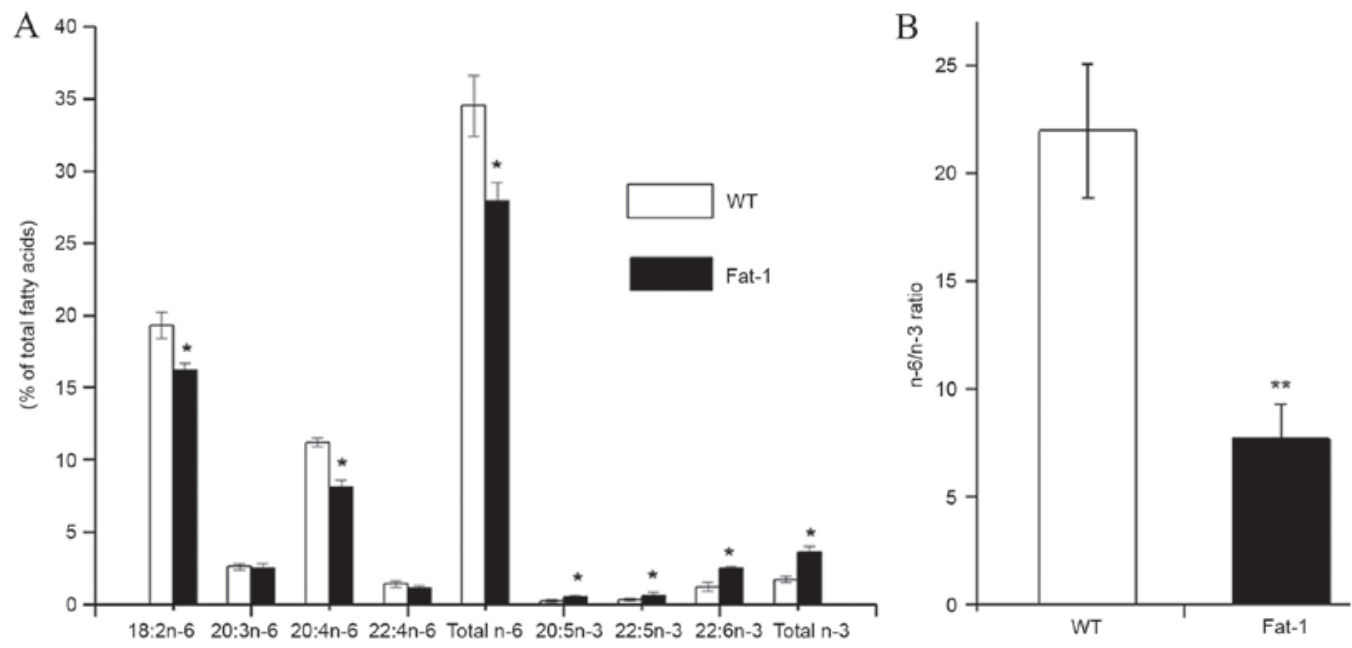

Figure 2. The n-3 and n-6 lipid profile of WT and fat-1 transgenic mouse B16-F10 melanoma tumor tissues using gas chromatography. (A) Quantitative analysis of the composition of $n-3$ and n- 6 fatty acids and (B) the ratio of n- $6 / n-3$ fatty acids in the melanoma tissues of WT and fat- 1 mice. Values represent the mean \pm standard error from three separate measurements, $\mathrm{n}=6 .{ }^{*} \mathrm{P}<0.05$ and ${ }^{* *} \mathrm{P}<0.01 \mathrm{vs.}$. WT controls. WT, wild-type; fat- 1 , omega-3 fatty acid desaturase; 18:2n-6, linoleic acid; 20:3n-6, dihomo-gamma-linolenic acid; 22:4n-6, adrenic acid; 20:5n-3, eicosapentaenoic acid; 22:5n-3, docosapentaenoic acid; 22:6n-3, docosahexaenoic acid.

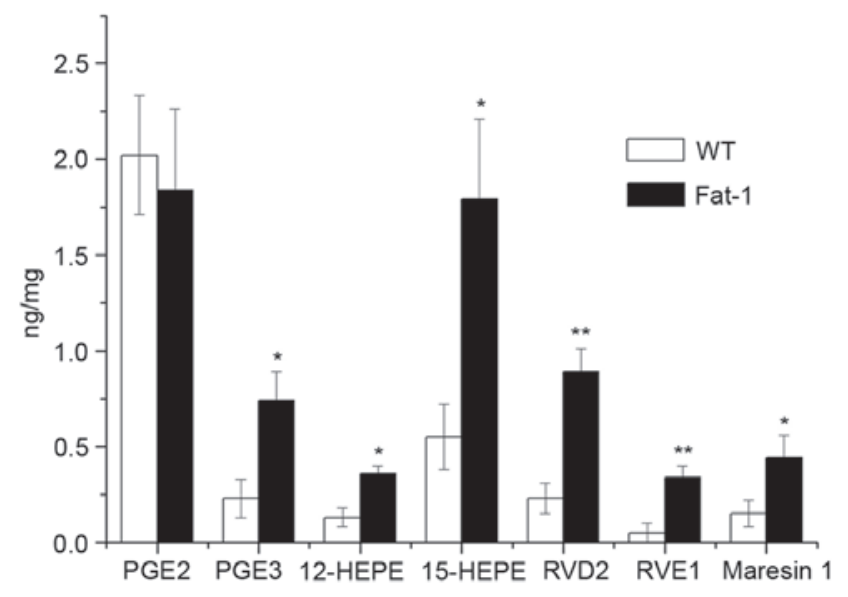

Figure 3. B16-F10 mouse melanoma tumors from transgenic fat-1 mice display altered lipid metabolite profiles. Ultra-performance liquid chromatography-ultraviolet-tandem mass spectrometry analysis of the arachidonic acid, eicosapentaenoic acid and docosahexaenoic acid lipid metabolites in mouse melanoma tumors from transgenic fat- 1 mice and WT controls. Values represent the mean \pm standard error. ${ }^{*} \mathrm{P}<0.05$ and ${ }^{* *} \mathrm{P}<0.01$ vs. WT controls. WT, wild-type; fat-1, omega-3 fatty acid desaturase; PGE, prostaglandin; HEPE, hydroxyeicosapentaenoic acid; RV, resolvin.

levels of these transcriptional repressors. As shown in Fig. 4, the protein expression levels of ZEB1 and Snail were markedly decreased in the tumors of fat- 1 mice compared with those of WT mice. In contrast, no significant alterations in the expression of Slug, ZEB2 and Twist proteins were observed (data not shown). The transcription factors NF- $\mathrm{kB}$ and STAT3 are activated in a range of human cancers and are thought to promote tumorigenesis, in part, through their ability to regulate the expression levels of E-cadherin transcriptional repressors (32). As shown in Fig. 4, fat-1 mouse tumor tissues exhibited a slight decrease in NF- $\kappa \mathrm{B}$ protein expression levels, but a marked decrease in p-NF-кB (Ser536), compared to those from WT mice. Similarly, despite observing no significant alterations in the expression levels of STAT3, p-STAT3 (Tyr705) expression

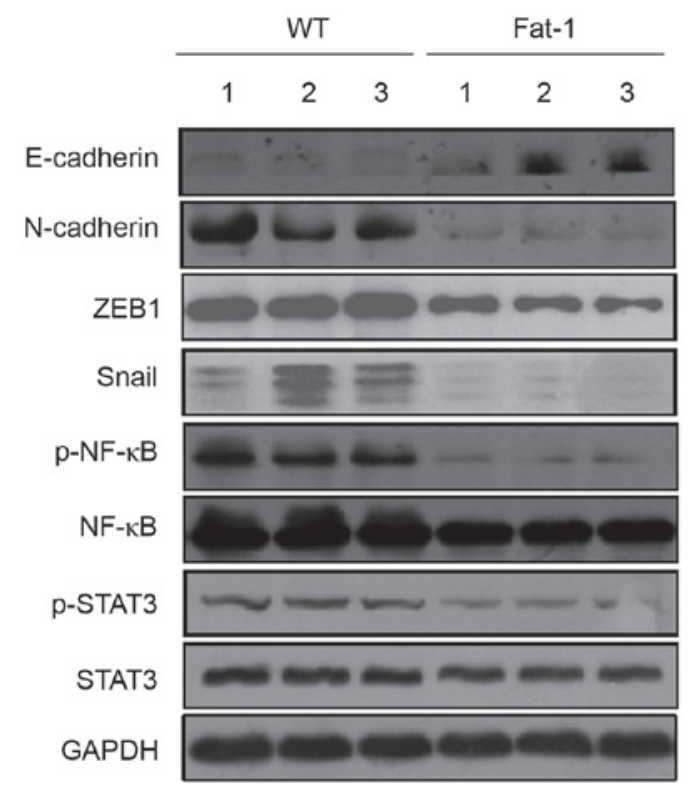

Figure 4. Endogenous n-3 PUFAs may upregulate E-cadherin expression by inhibiting the expression of its master regulators. Western blotting analysis

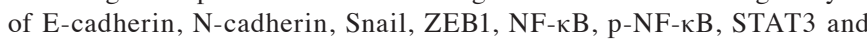
p-STAT3 protein expression levels in B16-F10 mouse melanoma tumor tissues from three WT (lanes 1-3) and three transgenic fat-1 mice (lanes 4-6). PUFAs, polyunsaturated fatty acids; ZEB1, zinc finger E-box 1; NF-кB, nuclear factor $\mathrm{\kappa B}$; STAT3, signal transducer and activator of transcription 3;

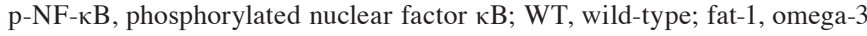
fatty acid desaturase.

was substantially decreased in the tumor tissues of fat- 1 mice. These results suggest that NF- $\mathrm{KB}$ and STAT3 may be involved in modulating E-cadherin expression.

Inhibition of EGFR/Akt/ $\beta$-catenin signaling pathway by $n-3$ PUFAs in fat- 1 transgenic mice. $\beta$-catenin functions as a transcriptional regulator in the canonical and noncanonical Wnt signaling pathways, and serves a critical role in regulating development, cell proliferation, differentiation and neoplastic 
transformation (10). To determine whether the low $n-6 / n-3$ PUFA ratio in transgenic fat- 1 mice affects $\beta$-catenin signaling pathways, western blotting was used to examine the protein expression levels of $\beta$-catenin and c-Myc; a known transcriptional target of $\beta$-catenin. As shown in Fig. 5, fat-1 tumor tissues exhibited a marked decrease in the expression levels of $\beta$-catenin and c-Myc compared with those from WT mice. $\beta$-catenin is activated, not only by Wnt ligands, but also by additional signaling pathways, such as the EGFR/Akt/GSK-3 $\beta$ pathway (33). Cytoplasmic $\beta$-catenin is controlled by the GSK-3 $\beta$-containing destruction complex. When GSK-3 $\beta$ is phosphorylated and inactivated, cytoplasmic $\beta$-catenin is stabilized and translocated to the nucleus (34). To determine whether $\beta$-catenin degradation may occur through inhibition of the phosphorylation of GSK-3 $\beta$ and upstream kinases, EGFR and Akt, the protein expression levels of GSK-3 $\beta$, p-GSK-3 $\beta$, EGFR, Akt and p-Akt were examined. As shown in Fig. 5 no significant differences in GSK-3 $\beta$ and Akt protein expression levels were observed in the melanoma tumor tissues of fat- 1 and WT mice, whereas the expression levels of p-GSK-3 $\beta$, p-Akt and EGFR were markedly decreased in fat-1 tissues compared with those of WT mice. This indicates that the EGFR/Akt/GSK-3 $\beta$ signaling pathway may be involved in controlling endogenous n-3 PUFA-induced $\beta$-catenin degradation.

Tumor growth inhibition was not associated with $n-3$ PUFA-induced oxidative stress in fat-1 mice. A lipid peroxidation assay was performed to determine lipid peroxidation levels in fat-1 and WT mice with or without Vit E administration. As shown in Fig. 6A, the urine from fat-1 mice demonstrated an increased level of MDA compared with that of WT mice (20.3 \pm 2.1 vs. $11.7 \pm 2.7 \mu \mathrm{M} ; \mathrm{P}=0.0293)$. Notably, no significant difference in the levels of MDA was observed between fat-1 and WT mice $(12.9 \pm 3.2$ vs. $8.3 \pm 1.8 \mu \mathrm{M})$ following the administration of Vit E. In addition, tumor growth was suppressed in fat- 1 and WT mice, and fat-1 mice exhibited decreased tumor growth compared with WT-mice following Vit E supplementation (Fig. 6B).

\section{Discussion}

Malignant melanoma is one of the most common life-threatening cancers among young western populations due to its rapid progression and high metastatic potential (35). The development of new therapies for malignant melanoma, including immunotherapy and targeted-molecular therapy, faces challenges due to the occurrence of drug resistance and harmful side effects (8). Notably, there is increasing evidence that natural products, such as dietary lipids, are considered to be of crucial importance in regulating melanoma cell growth (36). However, the molecular mechanisms underlying the role of $n-3$ PUFAs in melanoma development, progression and prevention are not fully understood. In the present study, to evaluate the antitumor effects of n-3 PUFAs on melanoma in vivo, B16-F10 mouse melanoma cells, which metastasize to the lungs, were injected into fat- 1 and WT mice. The fat- 1 transgenic mouse is a suitable experimental model as the mice can maintain a balanced n-6/n-3 fatty acid ratio in all organs and tissues, due to the ubiquitous expression of the Caenorhabditis elegans

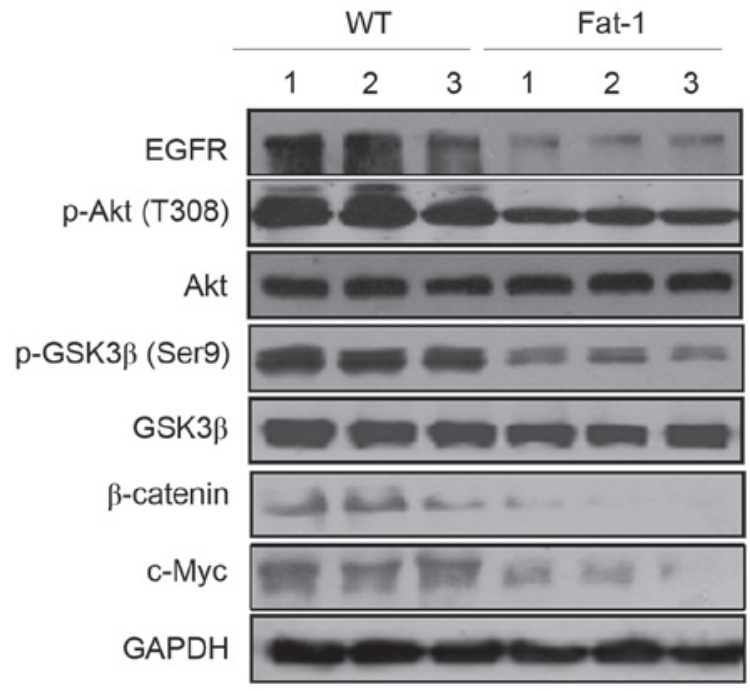

Figure 5. Endogenous n-3 PUFAs modulate $\beta$-catenin signaling. Western blotting analysis of $\beta$-catenin, c-Myc, EGFR and Akt, p-Akt, GSK-3 $\beta$ and p-GSK-3 $\beta$ protein expression levels in B16-F10 mouse melanoma tumor tissues from three WT (lanes 1-3) and three fat-1 transgenic mice (lanes 4-6). PUFAs, polyunsaturated fatty acids; EGFR, epidermal growth factor receptor; p-Akt, phosphorylated Akt; GSK-3 $\beta$, glycogen synthase kinase-3 $\beta$; WT, wild-type; fat-1, omega-3 fatty acid desaturase.

desaturase gene (26). The genetic approach presented in this study enabled the production of two different fatty acid profiles in tumor tissues whilst using identical diets rich in linoleic acid (18:2, n-6) but lacking in n-3 fatty acids. Although the formation of tumors was not significantly inhibited in fat-1 transgenic mice compared with those of WT mice, endogenous production of n-3 PUFAs in fat-1 mice markedly decreased the growth rate of the xenografts. Moreover, melanoma tumor growth inhibition in fat-1 mice was correlated with the production of n-3 PUFA-antitumor derivatives, the modulation of E-cadherin expression and inhibition of $\beta$-catenin signaling.

E-cadherin is an important adhesion molecule in epithelial cells that functions to mediate cell-to-cell adhesion and is a potent invasion/tumor suppressor (5). In the present study, B16-F10 melanoma tumors from fat-1 mice displayed a significant upregulation in the protein expression levels of the E-cadherin epithelial biomarker and a marked decrease in $\mathrm{N}$-cadherin expression (a marker of mesenchymal stage), compared with those of WT mice. This suggests that the reverse EMT process, known as mesenchymal-epithelial transition, may have occurred in fat-1 tumor tissues. Consistent with these results, recent studies have demonstrated that breast and colorectal cancer tissues from fat- 1 mice display increased levels of E-cadherin expression, which indicates that its expression may be modulated by endogenous n-3 PUFAs $(37,38)$. However, in the present study, the formation of metastases in the lungs or other organ sites in fat-1 and WT mice was not observed (data not shown). One possible reason for this is that the subcutaneous tumor model may not favor lung metastases of B16-F10 cells, compared with the intravenous injection tumor model. In addition, it is virtually impossible for cancer cells to disseminate from a primary tumor to a more distant tissue site within a period of 15 days (39). Therefore, further studies to investigate the anti-metastatic activities of n-3 PUFAs using the intravenous injection tumor model are 


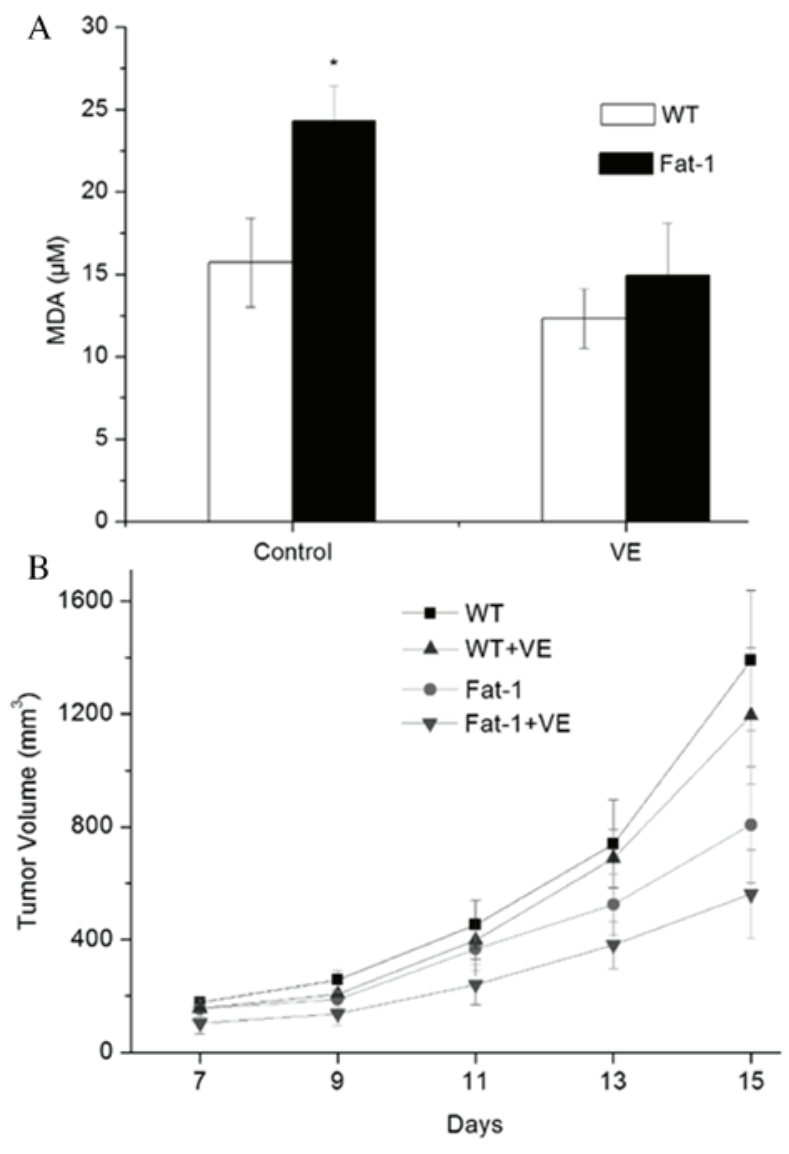

Figure 6. Vit E counteracts n-3 PUFA-induced lipid peroxidation and enhances the antitumor effects of n-3 PUFAs. (A) MDA concentrations in the urine of fat-1 and WT mice treated with or without Vit E. Results are presented as mean \pm standard error. ${ }^{*} \mathrm{P}<0.05$ vs. WT controls. (B) Mouse melanoma tumor volumes in WT and fat-1 transgenic mice following the administration of Vit E. Vit E, vitamin E; PUFA, polyunsaturated fatty acid MDA, malondialdehyde; fat-1, omega-3 fatty acid desaturase; WT, wild-type.

required. In the present study, the protein expression levels of the transcriptional repressors of E-cadherin, ZEB1 and Snail, were markedly decreased, suggesting that ZEB1 and Snail may have been involved in regulating E-cadherin expression in the fat-1 tumor tissues. Consistent with these results, Galindo-Hernandez et al (40) reported that the expression of Snail and Twist was correlated with linoleic acid (n-6 PUFAs) -mediated downregulation of E-cadherin expression in breast cancer cells. NF- $\kappa$ B and STAT3 transcription factors are principle regulators of inflammation, and have been implicated in the pathogenesis of immune disorders and cancer (41). The results of the present study demonstrated that the mouse melanoma tumor tissues from fat-1 mice exhibited lower levels of $\mathrm{p}-\mathrm{NF}-\kappa \mathrm{B}$ and $\mathrm{p}-\mathrm{STAT} 3$ protein expression compared with those of WT mice. NF- $\kappa \mathrm{B}$ and STAT3 have been identified as important regulators of EMT in several different types of cancer (42). Therefore, it is possible that NF- $\kappa$ B and STAT3 modulated E-cadherin expression in fat-1 mice, through regulating the transcriptional repressors of E-cadherin.

A notable finding of the present study was the observed inhibition of the $\beta$-catenin signaling pathway in fat- 1 mice. $\beta$-catenin, a key component of the Wnt signaling pathway and the cadherin/catenin-based adhesion process, serves an important role in the development of melanoma and its evasion of the immune system $(43,44)$. Therefore, we hypothesize that the observed disruption to the $\beta$-catenin signaling pathway may be attributed to the effective chemopreventative and antitumorigenic effects of n-3 PUFAs. Decreased tumor growth was associated with fat-1-mediated downregulation of $\beta$-catenin, p-GSK-3 $\beta$ and c-Myc protein expression levels. These results are consistent with those of other studies demonstrating that endogenous n-3 PUFAs were associated with a reduction in p-GSK-3 $\beta$ levels and a subsequent downregulation of $\beta$-catenin in prostate and pancreatic cancer tissues, which supports our hypothesis that the antitumor effects of n-3 PUFAs in melanoma may involve GSK-3 $\beta$-mediated $\beta$-catenin degradation $(45,46)$. In contrast, Castellone et al (47) demonstrated that PGE2, an AA (n-6 PUFA) -derived prostanoid, enhances the stability of $\beta$-catenin through promoting GSK-3 $\beta$ phosphorylation. n-3 PUFAs are readily incorporated into cell membranes and lipid rafts, and their incorporation may lead to changes in the levels of membrane-associated signaling proteins, including Ras, Akt, EGFR and human epidermal growth factor receptor 2 (48). In the present study and the study by Pai et al (49), the observed reductions in the expression levels of EGFR and p-Akt protein in fat-1 tumor tissues suggest that the EGFR/Akt signaling pathway may be involved in n-3 PUFA-mediated $\beta$-catenin degradation. In addition, increased E-cadherin expression may also account for the decreased protein expression levels of $\mathrm{c}-$ Myc through sequestering $\beta$-catenin in the plasma membrane, thereby blocking its translocation to the nucleus. Ultimately, the results of the present study suggest that $\beta$-catenin signaling inhibition contributes to the preventive effect of n-3 PUFAs against melanoma.

It is generally accepted that n-3 PUFAs exert their potentially beneficial anti-inflammatory effects by decreasing the levels of AA-derived mediators, such as the predominant proinflammatory prostanoids PGE2 and PGI2 (50). In the present study, no notable alterations in the levels of PGE2 between fat-1 and WT mice were observed. In contrast, marked differences in the levels of EPA metabolites (PGE3, 12-HEPE, 15-HEPE and RVE1) and DHA-derived mediators (RVD2 and Maresin1) were observed in the tumors of fat- 1 mice compared with those of WT mice. Preclinical and clinical trials have provided evidence to show that EPA and DHA-derived lipid mediators exert their potential anti-inflammatory effects in a wide range of inflammatory diseases, including arthritis, lung inflammation and colorectal cancer (51). The decreased expression levels of $\mathrm{p}-\mathrm{NF}-\kappa \mathrm{B}$ and $\mathrm{p}-\mathrm{STAT} 3$ in the melanoma tumors of fat- 1 mice in the present study, confirmed the anti-inflammatory effects of n-3 PUFA-derived mediators. Taking these findings into account, it is possible that the endogenously biosynthesized lipid mediators derived from n-3 PUFAs underlie the antitumor effects observed in the fat-1 transgenic mice, instead of the decrease in AA-derived mediators, such as 2-series prostaglandins (PGE2).

Oxidative stress has been reported to serve an important role in tumor initiation and progression (52). The excessive intracellular accumulation of reactive oxygen species leads to disruption of the mitochondrial membrane potential, the release of cytochrome c and ultimate cell apoptosis (53). DHA and EPA unsaturated fatty acids are susceptible to lipid peroxidation, which can lead to high levels of oxidative stress, cell growth inhibition and cell death (54). Indeed, in the present 
study, a significant increase in MDA levels was observed in the urine of fat-1 mice compared with that of WT mice. However, no significant alterations in the MDA levels between fat-1 and WT mice were observed following administration of the Vit E antioxidant, which suggests that the lipid peroxidation induced by endogenous PUFAs may be counteracted by Vit E. Notably, tumor growth was suppressed in fat-1 and WT mice following Vit E administration, and tumor growth inhibition was greater in fat-1 mice supplemented with Vit E compared with WT controls. Ultimately, these data suggest that the protective role of n-3 PUFAs against melanoma progression, may not be mediated by n-3 PUFA-induced lipid peroxidation, and demonstrates that Vit E supplementation may further enhance the antitumor activity of n-3 PUFAs.

In conclusion, using an in vivo model involving fat-1 transgenic mice, the present study provides encouraging preclinical evidence of the molecular mechanisms by which n-3 PUFAs may regulate the malignant features of melanoma. The results presented support a protective role of $n-3$ PUFAs in the prevention of melanoma progression, and in the potential development of clinical interventions that combine n-3 PUFAs with conventional or innovative therapies for the treatment of patients with melanoma.

\section{Acknowledgements}

This study was partly sponsored by the Fang Runhua Fund of Hong Kong, the K.C. Wong Magna Fund of Ningbo University, the National Science Foundation of China (grant nos. 81172660, 81450048, 281370165 and 31201284), the Scientific Innovation Team Project of Ningbo (grant no. 2014B82002), the Zhejiang Provincial Natural Science Foundation of China (grant nos. LY14H260001 and LY15C200008), the projects of the Medical and Health Technology Development Program of Zhejiang (grant no. 2014kya198), the Natural Science Foundation of Ningbo (grant nos. 2015A610222 and 2013A610209), the Science Foundation of Ningbo University (grant no. XKL14D2101) and the project of the Application of Public Welfare Technology (Experimental Animals) of Zhejiang (grant no. 2016C37120). We are also grateful to Mr An-Shi Wang (Ningbo University, Ningbo, China) for performing cell culture and Mr Fei Zhou (Ningbo University) for providing helpful advice.

\section{References}

1. American Cancer Society: Cancer Facts and Figures 2014 Atlanta, GA, American Cancer Society, 2014.

2. Jemal A, Siegel R, Ward E, Hao Y, Xu J and Thun MJ: Cancer statistics, 2009. CA Cancer J Clin 59: 225-249, 2009.

3. Kuphal S and Bosserhoff A: Recent progress in understanding the pathology of malignant melanoma. J Pathol 219: 400-409, 2009.

4. Flaherty KT, Lee SJ, Zhao F, Schuchter LM, Flaherty L, Kefford R, Atkins MB, Leming P and Kirkwood JM: Phase III trial of carboplatin and paclitaxel with or without sorafenib in metastatic melanoma. J Clin Oncol 31: 373-379, 2013.

5. van Roy F: Beyond E-cadherin: Roles of other cadherin superfamily members in cancer. Nat Rev Cancer 14: 121-134, 2014.

6. Schmalhofer O, Brabletz S and Brabletz T: E-cadherin, beta-catenin, and ZEB1 in malignant progression of cancer. Cancer Metastasis Rev 28: 151-166, 2009.

7. Heerboth S, Housman G, Leary M, Longacre M, Byler S, Lapinska K, Willbanks A and Sarkar S: EMT and tumor metastasis. Clin Transl Med 4: 6, 2015.
8. Sullivan RJ and Flaherty KT: Resistance to BRAF-targeted therapy in melanoma. Eur J Cancer 49: 1297-1304, 2013.

9. Clevers $\mathrm{H}$ and Nusse R: Wnt/ $/$-catenin signaling and disease. Cell 149: 1192-1205, 2012.

10. Nelson WJ and Nusse R: Convergence of Wnt, beta-catenin, and cadherin pathways. Science 303: 1483-1487, 2004.

11. Sinnberg T, Menzel M,Kaesler S, Biedermann T, Sauer B, Nahnsen S, Schwarz M, Garbe C and Schittek B: Suppression of casein kinase 1alpha in melanoma cells induces a switch in beta-catenin signaling to promote metastasis. Cancer Res 70: 6999-7009, 2010.

12. Lucero OM, Dawson DW, Moon RT and Chien AJ: A re-evaluation of the 'oncogenic' nature of Wnt/ $\beta$-catenin signaling in melanoma and other cancers. Curr Oncol Rep 12: 314-318, 2010.

13. Jones V and Katiyar SK: Emerging phytochemicals for prevention of melanoma invasion. Cancer Lett 335: 251-258, 2013.

14. Wu Y, Lin Y, Liu H and Li J: Inhibition of invasion and up-regulation of E-cadherin expression in human malignant melanoma cell line A375 by (-)-epigallocatechin-3-gallate. J Huazhong Univ Sci Technol Med Sci 28: 356-359, 2008.

15. Vaid M,Prasad R, Sun Q and Katiyar SK: Silymarin targets $\beta$-catenin signaling in blocking migration/invasion of human melanoma cells. PLoS One 6: e23000, 2011.

16. Serini S, Fasano E, Celleno L, Cittadini A and Calviello G: Potential of long-chain n-3 polyunsaturated fatty acids in melanoma prevention. Nutr Rev 72: 255-266, 2014.

17. Algamas-Dimantov A, Yehuda-Shnaidman E, Hertz R, Peri I, Bar-Tana J and Schwartz B: Prevention of diabetes-promoted colorectal cancer by (n-3) polyunsaturated fatty acids and (n-3) PUFA mimetic. Oncotarget 5: 9851-9863, 2014.

18. Denkins Y, Kempf D, Ferniz M, Nileshwar S and Marchetti D: Role of omega-3 polyunsaturated fatty acids on cyclooxygenase-2 metabolism in brain-metastatic melanoma. J Lipid Res 46: 1278-1284, 2005.

19. Miller AB and Gaudette LA: Cancers of skin, bone, connective tissues, brain, eye, thyroid and other specified and unspecified sites in Inuit. Acta Oncol 35: 607-616, 1996.

20. Bain C, Green A, Siskind V, Alexander J and Harvey P: Diet and melanoma. An exploratory case-control study. Ann Epidemiol 3: 235-238, 1993.

21. Gallagher RP, Elwood JM and Hill GB: Risk factors for cutaneous malignant melanoma: The Western Canada Melanoma Study. Cancer Res 102: 38-55, 1986

22. Osterlind A, Tucker MA, Stone BJ and Jensen OM: The Danish case-control study of cutaneous malignant melanoma. IV. No association with nutritional factors, alcohol, smoking or hair dyes. Int J Cancer 42: 825-828, 1988.

23. Kirkpatrick CS, White E and Lee JA: Case-control study of malignant melanoma in Washington State. II. Diet, alcohol, and obesity. Am J Epidemiol 139: 869-880, 1994.

24. Salem ML, Kishihara K, Abe K, Matsuzaki G and Nomoto K: N-3 polyunsaturated fatty acids accentuate B16 melanoma growth and metastasis through suppression of tumoricidal function of $\mathrm{T}$ cells and macrophages. Anticancer Res 20: 3195-3203, 2000.

25. Demchenko DV, Pozharitskaya ON, Shikov AN and Makarov VG: Validated HPTLC Method for Quantification of Vitamin D-3 in Fish Oil. Journal of Planar Chromatography 24: 487-490, 2011.

26. Kang JX, Wang J, Wu L and Kang ZB: Transgenic mice: Fat-1 mice convert n-6 to n-3 fatty acids. Nature 427: 504, 2004.

27. Xia S, Lu Y, Wang J, He C, Hong S, Serhan CN and Kang JX: Melanoma growth is reduced in fat-1 transgenic mice: Impact of omega-6/omega-3 essential fatty acids. Proc Natl Acad Sci USA 103: 12499-12504, 2006.

28. Bellenger J, Bellenger S, Clément L, Mandard S, Diot C, Poisson JP and Narce M: A new hypotensive polyunsaturated fatty acid dietary combination regulates oleic acid accumulation by suppression of stearoyl CoA desaturase 1 gene expression in the SHR model of genetic hypertension. FASEB J 18: 773-775, 2004.

29. Wang Y, Armando AM, Quehenberger O, Yan C and Dennis EA: Comprehensive ultra-performance liquid chromatographic separationand mass spectrometric analysis of eicosanoid metabolites in human samples. J Chromatogr A 1359: 60-69, 2014.

30. Serhan CN, Chiang N, Dalli J and Levy BD: Lipid mediators in the resolution of inflammation. Cold Spring Harb Perspect Biol 7: a016311, 2014

31. Thiery JP, Acloque H, Huang RY and Nieto MA: Epithelial- mesenchymal transitions in development and disease. Cell 139: 871-890, 2009.

32. Lin WL, Lai DY, Lee YJ, Chen NF and Tseng TH: Antitumor progression potential of morusin suppressing STAT3 and NFKB in human hepatoma SK-Hep1 cells. Toxico Lett 232: 490-498, 2015. 
33. Ge $\mathrm{C}$, Yu M and Zhang C: G protein-coupled receptor 30 mediates estrogen-induced proliferation of primordial germ cells via EGFR/Akt/ $\beta$-catenin signaling pathway. Endocrinology 153 : 3504-3516, 2012

34. Novak A and Dedhar S: Signaling through beta-catenin and Lef/Tcf. Cell Mol Life Sci 56: 523-537, 1999.

35. Siegel R, Ward E, Brawley O and Jemal A: Cancer statistics, 2011: The impact of eliminating socioeconomic and racial disparities on premature cancer deaths. CA Cancer J Clin 61: 212-236, 2011

36. Fortes C, Mastroeni S, Melchi F, Pilla MA, Antonelli G Camaioni D, Alotto $\mathrm{M}$ and Pasquini P: A protective effect of the Mediterranean diet for cutaneous melanoma. Int J Epidemiol 37: 1018-1029, 2008

37. Algamas-Dimantov A, Yehuda-Shnaidman E, Hertz R, Peri I, Bar-Tana J and Schwartz B: Prevention of diabetes-promoted colorectal cancer by (n-3) polyunsaturated fatty acids and (n-3) PUFA mimetic. Oncotarget 5: 9851-9863, 2014.

38. Algamas-Dimantov A, Davidovsky D, Ben-Ari J, Kang JX, Peri I, Hertz R, Bar-Tana J and Schwartz B: Amelioration of diabesity-induced colorectal ontogenesis by omega-3 fatty acids in mice. J Lipid Res 53: 1056-1070, 2012.

39. Simchuk EJ and Low DE: Direct esophageal metastasis from a distant primary tumor is a submucosal process: A review of six cases. Dis Esophagus 14: 247-250, 2001.

40. Galindo-Hernandez O, Serna-Marquez N, Castillo-Sanchez R and Salazar EP: Extracellular vesicles from MDA-MB-231 breast cancer cells stimulated with linoleic acid promote an EMT-like process in MCF10A cells. Prostaglandins Leukot Essent Fatty Acids 91: 299-310, 2014.

41. Bollrath J and Greten FR: IKK/NF-kappaB and STAT3 pathways: Central signalling hubs in inflammation-mediated tumour promotion and metastasis. EMBO Rep 10: 1314-1319, 2009.

42. Min C, Eddy SF, Sherr DH and Sonenshein GE: NF-kappaB and epithelial to mesenchymal transition of cancer. J Cell Biochem 104: 733-744, 2008.

43. Damsky WE, Curley DP, Santhanakrishnan M, Rosenbaum LE, Platt JT, Gould Rothberg BE, Taketo MM, Dankort D, Rimm DL, McMahon $\mathrm{M}$ and Bosenberg $\mathrm{M}$ : $\beta$-catenin signaling controls metastasis in Braf-activated Pten-deficient melanomas. Cancer Cell 20: 741-754, 2011.
44. Spranger S, Bao R and Gajewski TF: Melanoma-intrinsic $\beta$-catenin signalling prevents anti-tumor immunity. Nature 523: 231-235, 2015.

45. Lu Y, Nie D, Witt WT, Chen Q, Shen M, Xie H, Lai L, Dai Y and Zhang J: Expression of the fat-1 gene diminishes prostate cancer growth in vivo through enhancing apoptosis and inhibiting GSK-3 beta phosphorylation. Mol Cancer Ther 7: 3203-3211, 2008.

46. Song KS, Jing K, Kim JS, Yun EJ, Shin S, Seo KS, Park JH, Heo JY, Kang JX, Suh KS, et al: Omega-3-polyunsaturated fatty acids suppress pancreatic cancer cell growth in vitro and in vivo via downregulation of Wnt/Beta-catenin signaling. Pancreatology 11: 574-584, 2011.

47. Castellone MD, Teramoto H, Williams BO, Druey KM and Gutkind JS: Prostaglandin E2 promotes colon cancer cell growth through a Gs-axin-beta-catenin signaling axis. Science 310: 1504,2005

48. Biondo PD, Brindley DN, Sawyer MB and Field CJ: The potential for treatment with dietary long-chain polyunsaturated n-3 fatty acids during chemotherapy. J Nutr Biochem 12: 787-796, 2008.

49. Pai R, Nakamura T, Moon WS and Tarnawski AS: Prostaglandins promote colon cancer cell invasion; signaling by cross-talk between two distinct growth factor receptors. FASEB J 17: 1640-1647, 2003.

50. Calder PC: n-3 polyunsaturated fatty acids, inflammation, and inflammatory diseases. Am J Clin Nutr 83 (6 Suppl): 1505S-1519S, 2006.

51. Serhan CN: Pro-resolving lipid mediators are leads for resolution physiology. Nature 510: 92-101, 2014.

52. Lone AA, Ganai SA, Ahanger RA, Bhat HA, Bhat TA and Wani IA: Free radicals and antioxidants: Myths, facts and mysteries. African Journal of Pure and Applied Chemistry 7: 91-113, 2013

53. Kim KY, Cho HJ, Yu SN, Kim SH, Yu HS, Park YM, Mirkheshti N, Kim SY, Song CS, Chatterjee B and Ahn SC: Interplay of reactive oxygen species, intracellular $\mathrm{Ca} 2+$ and mitochondrial homeostasis in the apoptosis of prostate cancer cells by deoxypodophyllotoxin. J Cell Biochem 114: 1124-1134, 2013.

54. Azad MB, Chen Y and Gibson SB: Regulation of autophagy by reactive oxygen species (ROS): Implications for cancer progression and treatment. Antioxid Redox Signal 11: 777-790, 2009. 